\title{
HOMOMORPHISMS OF DISTRIBUTIVE P-ALGEBRAS
}

\section{WITH COUNTABLY MANY MINIMAL PRIME IDEALS}

\author{
M.E, Adams, V. Koubek and J. Sichler
}

\begin{abstract}
According to a result of Lee, varieties of pseudocomplemented distributive lattices form an $\omega+1$ chain $\underset{\sim}{B}-1 \subset \underset{\sim}{B} \subset \underset{\sim}{B} \subset \ldots \subset \underset{\sim}{B}$ in which $\underset{\sim}{B}$ is the trivial variety and $\underset{\sim}{\stackrel{B}{0}}$ is the variety of Boolean algebras. In the present paper it is shown that the variety $\underset{\sim}{B}$ contains an almost universal subcategory $\underset{\sim}{B}$ in which the members of $\operatorname{Hom}\left(B, B^{\prime}\right)$ associated with minimal prime ideals of $B$ form a countably infinite set for any $B, B^{\prime} \in \underset{\sim}{B}$. In particular, $B_{3}$ contains arbitrarily large algebras whose nontrivial endomorphisms form the countably infinite right zero semigroup. Our earlier results concerning categorical properties of varieties of pseudocomplemented distributive lattices show that no further reduction of the right zero count is possible.
\end{abstract}

Received 6 June 1986. The support of NSERC is gratefully acknowledged.

Copyright Clearance Centre, Inc, Serial-fee code: 0004-9729/87 $\$ A 2.00+0.00$. 


\section{Introduction.}

A pseudocomplemented distributive lattice or a $p$-algebra is an algebra $(L ; \vee, \wedge, *, 0,1)$ of type $(2,2,1,0,0)$ such that $(L ; \vee, \wedge, 0,1)$ is a distributive $(0,1)$-lattice equipped with an additional unary operation * of pseudocomplementation defined by the requirement that $y \leq x^{*}$ if and only if $x \wedge y=0$ in the lattice $(L ; \vee, \wedge, 0,1)$. According to Ribenboim [12], pseudocomplemented distributive lattices form a variety ${\underset{\sim}{B}}_{\omega}$. The lattice of subvarieties of $\underset{\sim}{B}$ was shown, in Lee [6], to be the countable chain $\underset{\sim}{B}{ }_{-1} \subset \underset{\sim}{B} \subset \underset{\sim}{B} \subset \ldots \subset \underset{\sim}{B} \underset{\omega}{ }$ in which $\underset{\sim}{B}-1$ is the trivial variety, $\underset{\sim}{B} 0$ consists of Boolean algebras, and $\underset{\sim}{B} \underset{1}{ }$ is the variety of stone algebras.

These varieties present an interesting spectrum of interdependence between the complexity of their algebras and their relative richness as categories. For instance, a stone algebra $S$ is uniquely determined within $\stackrel{B}{\sim}_{1}$ by its abstract endomorphism monoid End(S), and $\underset{\sim}{B_{2}}$ contains at most two nonisomorphic algebras with isomorphic endomorphism monoids [1].

On the other hand, for every infinite cardinal $k$ the variety ${\underset{\sim}{B}}_{3}$ contains $2^{k}$ (the maximal possible number) of paixwise nonisomorphic algebras whose endomorphism monoids are isomorphic [1], while in the variety ${\underset{\sim}{G}}_{4}$ a proper class of algebras with isomorphic endomorphism monoids can be found [2]. These earlier results suggest that $\underset{\sim}{B_{3}}$ is the variety most suitable for further investigations.

The present note shows that the categorical structure of ${\underset{\sim}{3}}_{3}$ is considerably richer than that of $\underset{\sim}{B_{2}}$. To formulate the main result, Theorem 1.1 below, the following observations are in order.

For any minimal prime ideal $I$ of a $p$-algebra $A$, the mapping $h: A \rightarrow\{0,1\}$ given by $h^{-1}\{0\}=I$ is a p-algebra homomorphism [3]. since any $p$-algebra $A$ has minimal prime ideals, there always are such homomorphisms between any two of them, and the endomorphism monoid End $(A)$ has at least one right zero. In fact, the submonoid of all right zeros of $\operatorname{End}(A)$ is in a one-to-one correspondence with the set of all 
minimal prime ideals of $A$.

Throughout the note, a homomorphism $g: A \rightarrow B$ is if $g(A)=\{0,1\} \subseteq B$.

A category $\underset{\sim}{ }$ is universal if every full category of algebras is isomorphic to a full subcategory of $\underset{\sim}{C}$. One of the consequences of universality (see Pultr and Trnkova [11]), is the existence of a proper class of nonisomorphic rigid objects, that is, objects whose endomorphism monoids consist of the identity endomorphism alone. Because of the existence of constant endomorphisms, p-algebras do not form a universal category.

Apart from the minimal prime ideal count, constant homomorphisms $h: A \rightarrow B$ do not reflect any of the structural complexity of $A$. To investigate the categorical richness of varieties of $p$-algebras more meaningfully, almost universal categories of $p$-algebras were defined in [1] as categories containing a class $\underset{\sim}{D}$ of objects such that all nonconstant homomorphisms between members of $\underset{\sim}{D}$ form a universal category. Observe that almost universality requires that non-constant homomorphisms never compose to a constant one.

The variety ${\underset{\sim}{3}}_{3}$ is the smallest almost universal variety of $p-$ algebras [1]. When of infinite cardinality $k$, the $p$-algebras constructed in [1] have $2^{k}$ minimal prime ideals and hence $2^{k}$ right zero endomorphisms, so that only a set of these algebras can have isomorphic endomorphism monoids. Attempting to reduce the right zero count, [2] exhibits a proper class of algebras in $\underset{\sim}{B_{3}}$ whose endomorphism monoids are finite; on the other hand, [2] shows that the requirement of only finitely many right zeros causes a loss of control over non-constant endomorphisms. Stated more precisely, the latter result says that any infinite almost rigid $p$-algebra in $\underset{\sim}{B}$ (that is, an algebra whose only non-constant endomorphism is the identity) must have infinitely many minimal prime ideals. The present note complements [2] by demonstrating that a class of algebras in $\underset{\sim 3}{B}$ whose set of minimal prime ideals is countably infinite determines an almost universal category.

THEOREM 1.1. The variety $\underset{\sim}{B}$ contains con almost universal subcategory $\underset{\sim}{B}$ in which the constant members of $\operatorname{Hom}\left(B, B^{\prime}\right)$ form $a$ 
countably infinite set for any $B, B^{\prime}$ in $\underset{\sim}{B}$.

In particular, $\underset{\sim}{B_{3}}$ contains a proper class of algebras whose endomorphism monoids are isomorphic to the countable right zero monoid. It may be of interest to note that the variety ${\underset{\sim}{B}}_{4}$ satisfies the variant of Theorem 1.1 with "countably infinite set" replaced by "an m-element finite set" [2].

The proof of Theorem 1.1 will be carried out using Priestley's duality [8] whose relevant features we briefly review first. The reader is referred to Davey and Duffus [4] or Priestley [10] for more detailed information.

A mapping $f: P \rightarrow P^{\prime}$ between posets $P, P^{\prime}$ is order preserving if $x \leq y$ in $P$ implies $f(x) \leq f(y)$ in $P^{\prime}$. For any subset $S$ of $P$ set $[S)=\{x \in P:$ B $s \in S x \geq s\}$ and $(S]=\{x \in P: \mathbb{H} s \in S x \leq s\}$; a subset $S$ of $P$ is increasing if $[S)=S$, decreasing when $(S]=S$. If Min(P) denotes the set of all minimal elements of $P$, then $\operatorname{Min}(S)=\operatorname{Min}(P) \cap(S]$ for any $S \subseteq P$; when $S=\{x\}$, we write $\operatorname{Min}(x)$ instead of $\operatorname{Min}(\{x\})$. A poset $P$ equipped with a topology $\tau$ is totally order disconnected if for any $x, y \in P$ with $x \$ y$ there exists a $\tau$-clopen decreasing set $S \subseteq P$ such that $y \in S$ and $x \in P \backslash S$.

PROPOSITION 1.2. (Priestley [8]). The category $\underset{\sim}{D}$ of all $(0,1)-$ homomorphisms of distributive $(0,1)$-lattices is dually isomorphic to the category $\underset{\sim}{T}$ of all continuous order preserving mappings of compact totally order disconnected spaces (henceforth called Priestley spaces).

The priestley space $(X, \tau, \leq)$ dual to a distributive lattice $D$ consists of the inclusion ordered prime ideals of $D$, so that $\operatorname{Min}(x)$ is nonvoid for every $x \in X$. Conversely, elements of the lattice $D$ are represented by clopen decreasing subsets of $X$. The dual of a $(0,1)$ lattice homomorphism $h: D^{\prime} \rightarrow D$ is the $\underset{\sim}{T}$-morphism $f$ such that, for any prime ideal $I$ of $D$ (that is, an element of the Priestley space $(X, \tau, \leq)$ dual to $D), f(I) \in X$ is the prime ideal $h^{-1}(I)$ of $D^{\prime}$.

A Priestley space $(X, \tau, \leq)$ is the dual of a $p$-algebra if and only if $[S)$ is clopen for every clopen decreasing $S \subseteq X$; such an ordered space will be called a p-space. The dual $f$ of a $p$-algebra homomorphism, called a $p$-map, is a continuous order preserving mapping 
such that $f(\operatorname{Min}(x))=\operatorname{Min}(f(x))$ for all elements $x$ of its domain (see priestley [9]). The constant homomorphisms of $p$-algebras defined earlier are thus represented by constant $p$-maps whose value is a minimal element of the appropriate $p$-space.

According to Lee [6], a p-algebra $A$ lies in the variety ${\underset{\sim}{3}}_{3}$ if and only if every prime ideal of $A$ contains at most three minimal prime ideals. We summarise these remarks as follows.

PROPOSITION 1.3 (Priestley [9] and Lee [6]). The variety ${\underset{\sim}{3}}_{3}$ of pseudocomplemented distributive lattices is dually isomorphic to the category of all p-spaces $(X, \tau, \leq)$ satisfying $|\operatorname{Min}(x)| \leq 3$ for all $x \in X$, and of all continuous order preserving moppings $f$ between such spaces such that $f(\operatorname{Min}(x))=\operatorname{Min}(f(x))$ for all $x$ in the domain space of $f$.

\section{The construction.}

If $S=(X, \tau, \leq)$ is a $p$-space representing an algebra from $\underset{\sim}{B_{3}}$ then $\operatorname{Min}(x)$ has at most three elements for every $x \in X$. The $p$-spaces constructed below to prove Theorem 1.1 consist of their minimal elements and of elements $x$ for which $\operatorname{Min}(x)$ has exactly three members. For any $p$-map $f: S \rightarrow S^{\prime}$ of such spaces, Proposition 1.3 shows that the $f$-image of any triple $\{a, b, c\}=\operatorname{Min}(x)$ for which there exists an $x \in S \backslash \operatorname{Min}(S)$ with $\operatorname{Min}(x)=\{a, b, c\}$ is either a singleton contained in $\operatorname{Min}\left(S^{\prime}\right)$ or the three-element subset $\{f(a), f(b), f(c)\}=\operatorname{Min}(f(x))$ of $\operatorname{Min}\left(S^{\prime}\right)$. The restriction of $f$ to $\operatorname{Min}(S)$ is thus a $t$-map (see the definition below).

The initial step describes the countable set $M=\operatorname{Min}(S)$ common to all spaces $S$ to be constructed, and the set $T$ of all triples $\{a, b, c\} \subseteq M$ for which each $S$ contains an $x$ with $\operatorname{Min}(x)=\{a, b, c\}$.

The set $M$ is the disjoint union of $A=\left\{a_{j}: j \in \omega\right\}, B=\left\{b_{j}: j \in 22\right\}$, and $C=\left\{c_{j}: j \in 3\right\}$, while $T$ is the union of the following sets:

$$
\begin{gathered}
\left\{\left\{b_{1}, b_{5}, b_{13}\right\},\left\{c_{0}, c_{1}, c_{2}\right\}\right\}, \\
T_{0}=\left\{\left\{a_{2 i}, a_{2 i+1}, a_{2 i+2}\right\}: i \in \omega\right\} \cup\left\{\left\{a_{1}, a_{3}, a_{5}\right\}\right\},
\end{gathered}
$$




$$
\begin{aligned}
& T_{1}=\left\{\left\{a_{2 i}, a_{2 i+5}, a_{2 i+10}\right\}: i \in \omega\right\} \cup\left\{\left\{a_{2}, a_{5}, a_{8}\right\}\right\}, \\
& U_{0}=\left\{\left\{b_{2 i-1}, b_{2 i}, b_{2 i+1}\right\}: i \in 22\right\}, \\
& U_{1}=\left\{\left\{b_{2 i}, a_{j}, b_{2 i+2 j+3}\right\}: i \in 22, j \in 4\right\} ;
\end{aligned}
$$

the addition of indices for members $b_{k}$ of $B$ is carried out in the arithmetic modulo 22 .

It is easy to verify that $(M, T)$ is a partial steiner triple system (see [7]) in that it satisfies:

(*) for every pair $\{x, y\} \subseteq M$ there exists at most one triple $\{x, y, z\} \in T$.

We say that $f:(M, T) \rightarrow\left(M^{P}, T^{\prime}\right)$ is a $t$-mop if $f(M) \subseteq M^{P}$ and if the $f$-image of every triple $\{x, y, z\} \in T$ is either a singleton or a triple in $T^{\prime}$. Hence if $t_{i}=\left\{x_{i}, y_{i}, z_{i}\right\} \in T$ for $i \in\{0,1\}$, then $f\left(z_{0}\right)=f\left(z_{1}\right)$ follows from $\left\{f\left(x_{0}\right), f\left(y_{0}\right)\right\}=\left\{f\left(x_{1}\right), f\left(y_{1}\right)\right\}$ for any $t-$ map $f$ into a triple system $\left(M^{0}, T^{p}\right)$ in which $\left(^{*}\right)$ holds; in what follows, this observation is frequently used without further reference.

First we aim to show that the only nonconstant $t$-map $f:(M \backslash C, T \backslash\{C\}) \rightarrow(M, T)$ is the inclusion of $M \backslash C$ into $M$. To this end, set $D=\left\{a_{0}, a_{1}, a_{2}, a_{3}\right\}$, and let $\sim$ denote the kernel of $f$.

LEMMA 2.1. If $f\left(b_{k}\right)=f\left(b_{k+i}\right)$ for some $k \in 22$ and $i \in\{1,2\}$ then $f$ is constant on $B \cup D$.

Proof. The pair $\left\{b_{k}, b_{k+i}\right\}$ collapsed by $f$ is contained in a triple from $U_{0}$ unless $k$ is even and $i=2 ;$ since $U_{1}$ includes triples $\left\{b_{k}, a_{0}, b_{k+3}\right\},\left\{b_{k+i}, a_{0}, b_{k+i+3}\right\}$, in the latter case $b_{k} \sim b_{k+i}$ implies $b_{k+3} \sim b_{k+i+3}$. Either way, $f$ collapses some triple $\left\{b_{s}, b_{s+1}, b_{s+2}\right\} \in U_{0}$. The existence, in $U_{1}$, of triples $\left\{b_{s-3}, a_{0}, b_{s}\right\}$ and $\left\{b_{s-3}, a_{1}, b_{s+2}\right\}$ now implies $a_{0} \sim a_{1}$. Using $\left\{b_{r}, a_{0}, b_{p+3}\right\}$, $\left\{b_{p}, a_{1}, b_{2+5}\right\}$ with even $r \in 22$ we see that $f$ collapses all members of $U_{0}$; thus $f$ is constant on $B$. Since each $a_{i} \in D$ lies in a triple whose other two elements are in $B$, we conclude that $f$ is constant on $B \cup D$. 
LEMMA 2.2. If $f\left(b_{r}\right)=f\left(b_{s}\right)$ with $r$ even and $s$ add then $f$ is constant on $B \cup D$.

Proof. There exists some $k \in 11$ such that $s=p+2 k+3$. If $k \in\{9,10\}$ then Lemma 2.1 gives the desired conclusion, so that it suffices to consider $k \in\{0, \ldots, 8\}$.

For any $j \in 4$ set $r(j)=s-(2 j+3)$ and $s(j)=r+2 j+3$; thus $s(j)-r(j)=r-s+2(2 j+3)=2(2 j-k)+3$. For each $k \in\{2, \ldots, 8\}$ there exists a $j \in 4$ with $2 j \in\{k-2, k-1\}$ and, therefore $s(j)-r(j) \in\{-1,1\}$. since $\left\{b_{r(j)}, a_{j}, b_{s}\right\},\left\{b_{p}, a_{j}, b_{s(j)}\right\}$ are triples in $U_{1}$, from $b_{r} \sim b_{s}$ it follows that $b_{r(j)} \sim b_{s(j)}$ for each $j \in 4$. Thus, by Lerma $2.1, f$ is constant on $B \cup D$.

If $k=0$ then $s=r+3$ and $f$ collapses the triple $\left\{b_{r}, a_{0}, b_{s}\right\}$; in particular, $a_{0} \sim b_{s}$. Applying this fact to $\left\{b_{s-1}, a_{0}, b_{s+2}\right\}$ and $\left\{b_{s}, b_{s+1}, b_{s+2}\right\}$, we obtain $b_{s-1} \sim b_{s+1}$. Hence $f$ is constant on $B \cup D$ by Lemma 2.1 .

For $k=1$ we have $s=r+5$, so that $b_{p} \sim b_{p+5}$. Using triples $\left\{b_{r}, a_{0}, b_{p+3}\right\}$ and $\left\{b_{p+3}, b_{n+4}, b_{p+5}\right\}$ now yields $a_{0} \sim b_{p+4}$. From $\left\{b_{p+4}, a_{0}, b_{n+7}\right\} \in U_{1}, b_{n+4} \sim b_{n+7}$ is obtained, and hence the previous case of $k=0$ applies again.

LEMMA 2.3. If $f\left(b_{p}\right)=f\left(b_{s}\right)$ for distinct elements of $B$ with $s-r$ even, then $f$ is constant on $B \cup D$.

Proof. For $r, s$ odd, the use of $\left\{b_{r-3}, a_{0}, b_{r}\right\}$ and $\left\{b_{s-3}, a_{0}, b_{s}\right\}$ shows that $b_{r-3} \sim b_{s-3}$; with no loss of generality we may thus assume. that $x, s$ are even.

From $\left\{b_{r}, a_{1}, b_{p+5}\right\},\left\{b_{s}, a_{1}, b_{s+5}\right\} \in U_{1}$ it now follows that $b_{p+5} \sim b_{s+5}$ which, in turn, yields $b_{p+2} \sim b_{s+2}$ since $\left\{b_{p+2}, a_{0}, b_{p+5}\right\}$ and $\left\{b_{s+2}, a_{0}, b_{s+5}\right\}$ are triples in $U_{1}$. Now $b_{r+2 i} \sim b_{s+2 i}$ follows inductively for all $i \in 11$. Since $b_{x} \neq b_{s}$, the least nonzero integer $j$ for which $b_{0} \sim b_{2 j}$ is less than 11 , so that $2 k_{j} \equiv: 2(\bmod 22)$ for 
some $k$. Clearly $b_{0} \sim b_{2 i j}$ for all $i$; in particular, $b_{0} \sim b_{2 k j}=b_{2}$ which, in view of 2.1 , completes the proof.

LEMMA 2.4. The restriction of $f$ to $B \cup D$ is either one-to-one or constant.

Proof. As seen above, $f$ is either one-to-one on $B$ or constant on $B \cup D$.

Should $f\left(a_{i}\right)=f\left(a_{j}\right)$ for distinct $a_{i}, a_{j} \in D$ then $f\left(b_{2 i+3}\right)=f\left(b_{2 j+3}\right)$ since $U_{1}$ contains triples $\left\{b_{0}, a_{i}, b_{2 i+3}\right\}$, $\left\{b_{0}, a_{j}, b_{2 j+3}\right\}$; thus $f$ is constant on $B \cup D$. The same conclusion follows when $f\left(a_{i}\right)=f\left(b_{j}\right)$ because of the existence of a triple $\left\{b_{j}, a_{i}, b_{k}\right\} \in U_{1}$ for any $i \in 4$ and $j \in 22$.

PROPOSITION 2.5. If $f:(M C, T \backslash\{C\})+(M, T)$ is a non-constant $t$-mop then $f(m)=m$ for all $m \in M C$.

Proof. For any $m \in M$ let $\operatorname{deg}(m)$, the degree of $m$, be the number of triples in $T$ that contain $m$. It is easy to see that at least eleven triples containing a given $\alpha_{i} \in D$ are contained in $B \cup D$, and that $\operatorname{deg}\left(a_{i}\right) \leq 4$ for all $a_{i} \in A \backslash D, 5 \leq \operatorname{deg}\left(b_{j}\right) \leq 7$ for all $j \in 22$, and $\operatorname{deg}\left(c_{k}\right)=1$ for $k \in 3$.

Suppose that $f$ is one-to-one on $B \cup D$. Since each $a_{i} \in D$ has degree at least eleven in $B \cup D$ and because all elements outside $D$ are of a smaller degree, $f(D)=D$. Since $f$ is one-to-one on $B \cup D$ and all elements outside $B \cup D$ are of degree less than five, it also follows that $f(B)=B$. Thus $f$ permutes the triples entirely contained in $B$; observe that $b_{1}, b_{5}, b_{13}$ are of degree 3 in $B$, that all other $b_{2 i+1}$ have $B$-degree two, and that each $b_{2 i}$ lies in a single triple contained in $B$. Since $f$ preserves $B$-degrees, it permutes each of the sets $E=\left\{b_{2 i}: i \in 11\right\}, F=\left\{b_{2 i+1}: i \neq 0,2,6\right\}$, and $G=\left\{b_{1}, b_{5}, b_{13}\right\}$. The images $f\left(t_{1}\right), f\left(t_{3}\right)$ of $B$-triples $t_{1}=\left\{b_{1}, b_{2}, b_{3}\right\}, t_{3}=\left\{b_{3}, b_{4}, b_{5}\right\}$ intersect in a member of $F$ and contain one element from each of $E, F$, and $G$. Since $t_{1}, t_{3}$ is the only pair of $B$-triples with these 
properties, $\left\{f\left(t_{1}\right), f\left(t_{3}\right)\right\}=\left\{t_{1}, t_{3}\right\}$; in particular, $f$ preserves $\left\{b_{1}, b_{5}\right\}$, and $f\left(b_{13}\right)=b_{13}$ since $\left\{b_{1}, b_{5}, b_{13}\right\} \in T$. similarly, the quadruple of consecutive $B$-triples $t_{i}=\left\{b_{i}, b_{i+1}, b_{i+2}\right\}$ with $i=5,7,9,11$ connects $b_{5}$ to $b_{13}$ through elements outside of $G$, while there is no such quadruple connecting $b_{1}$ and $b_{13}$. Therefore $f$ is the identity mapping on $G$ and, consequently, also on the union $\left\{b_{1}, \ldots, b_{13}\right\}$ of the six triples considered. It now easily follows that $f$ is the identity mapping on $B$.

Using triples $\left\{b_{0}, a_{j}, b_{2 j+3}\right\}$ with $j \in 4$ and the fact that $f\left(b_{k}\right)=b_{k}$ for all $k \in 22$ shows that $f$ maps $D=\left\{a_{0}, a_{1}, a_{2}, a_{3}\right\}$ identically onto itself.

Altogether, the $t$-map $f$ is either constant or the identity on $B \cup D$. To complete the proof we need to determine how $f$ extends to $A$. We set $A_{k}=\left\{a_{0}, \ldots, a_{2 k}\right\}$ and proceed inductively for $k \geq 4$.

Since $\left\{a_{2}, a_{3}, a_{4}\right\}$ and $\left\{a_{1}, a_{3}, a_{5}\right\}$ are triples in $T_{0}$ and because $f$ is a $t$-map into a triple system satisfying $(*)$, the values $f\left(a_{4}\right)$ and $f\left(a_{5}\right)$ are uniquely determined by $f+D:$ if $f\left(a_{i}\right)=v$ for all $a_{i} \in D$ then $f\left(a_{4}\right)=f\left(a_{5}\right)=v$, while $f\left(a_{i}\right)=a_{i}$ for $i=4,5$ follows when $f+D$ is the identity. Thus $f$ is either constant or the identity on $B \cup\left\{a_{0}, \ldots, a_{5}\right\}$. Using $\left\{a_{4}, a_{5}, a_{6}\right\} \in T_{0}$ and $\left\{a_{2}, a_{5}, a_{8}\right\} \in T_{1}$, in the first case we obtain $f\left(a_{6}\right)=v=f\left(a_{8}\right)$ and hence also $f\left(a_{7}\right)=v$ since $\left\{a_{6}, a_{7}, a_{8}\right\} \in T_{0}$, so that $f$ is constant on $B \cup A_{4}$. If, on the other. hand, $f$ is the identity on $B \cup\left\{a_{0}, \ldots, a_{5}\right\}$ then these three triples guarantee that $f$ is the identity also on $B \cup A_{4}$.

Assume that $f$ is either constant or the identity on some $B \cup A_{k}$ with $k \geq 4$. If $f(z)=v$ for all $z \in B \cup A_{k}$ then $f\left(a_{2 k+2}\right)=v$ since $f$ collapses the triple $\left\{a_{2 k-8}, a_{2 k-3}, a_{2 k+2}\right\} \in T_{1}$, and $f\left(a_{2 k+1}\right)=v$ now follows when $\left\{a_{2 k}, a_{2 k+1}, a_{2 k+2}\right\} \in T_{0}$ is used; hence $f$ is constant on 
$B \cup A_{k+1}$. If $f$ is the identity on $B \cup A_{k}$ then $f\left(a_{2 k+2}\right)=a_{2 k+2}$ follows from $(*)$ applied to the first triple, and a subsequent application of $(*)$ to the second one shows that $f$ is the identity $c \Omega$ $B \cup A_{k+1}$

Let $\underset{\sim}{\stackrel{T}{\sim}}$ be the category whose objects $W$ have the form $W=\left(Y, \sigma, s, y_{0}, y_{1}\right)$ for some compact totally order disconnected space $(Y, \sigma, \leq)$ and $y_{0}, y_{1} \in Y$. The morphisms of $\underset{\sim 2}{T}$ are all order preserving continuous mappings that also preserve each of the two distinguished elements. To prove Theorem 1.1, a full embedding $\Phi$ of the dually universal category $\underset{\sim}{\stackrel{T}{2}}$, (see [5]) into the category dual to that of all nonconstant homomorphisms between algebras from ${\underset{\sim}{B}}_{3}$ will be constructed as follows.

We set $\Phi(W)=\Phi\left(Y, \sigma, \leq, y_{0}, y_{1}\right)=(X, \tau, \leq)=S$, where $X$ is the disjoint union $Y \cup M \cup(T \backslash\{C\})$ for the triple system $(M, T)$ defined earlier. The partial order $\leq$ on $X$ is the extension of $(Y, S)$ defined by

$$
\begin{aligned}
& c \leq y \text { for all } c \in C \text { and all } y \in Y, \\
& m \leq t \text { for all } m \in M C \text { and all } t \in T \text { with } m \in t .
\end{aligned}
$$

It is clear that $M=\operatorname{Min}(S)$ and that $\operatorname{Min}(x)$ has exactly three elements for every $x \in X \backslash M$.

Set $A_{i}=\left\{a_{3 k+i} \in A: k \in \omega\right\}$ for $i=0,1,2$, and let $Z$ be a $\tau$-clopen set just when it satisfies

(a) if $i \in 3$ and $Z \cap A_{i}$ is infinite then $c_{i} \in Z$,

(b) if $i \in 3$ and $c_{i} \in Z$ then $A_{i} \backslash Z$ is finite,

(c) if $j \in 2$ and $Z \cap T_{j}$ is infinite then $y_{j} \in Z$,

(d) if $j \in 2$ and $y_{j} \in Z$ then $T_{j} \backslash Z$ is finite,

(e) $Z \cap Y$ is $\sigma$-clopen.

The singletons $\left\{c_{i}\right\},\left\{y_{j}\right\}$ thus compactify the respective discrete subspaces $A_{i}$ for $i \in 3$ and $T_{j}$ for $j \in 2$. Being the union of the compact space $W$ with these five compact spaces, $\Phi(W)$ is compact. 
To see that $\Phi(W)$ is totally order disconnected, choose $u \neq v$ in $X$ arbitrarily. Since the subspace $X \backslash(Y \cup C)$ is discrete, and $(x],[x)$ are finite for any $x \in X \backslash(Y \cup C)$, it suffices to consider the case of $u \in Y$ and $v \in Y \cup C$. If $v=c_{i}$ for some $i \in 3$ then $A_{i} \cup\{v\}$ is a $\tau-$ clopen decreasing set not containing $u$. Finally, let $u, v \in Y$. Then $v \in V$ and $u \in Y \backslash V$ for some $\sigma$-clopen decreasing subset $V$ of $Y$. If $J=\left\{j \in 2: y_{j} \in V\right\}$ then $\cup\left(T_{j}: j \in J\right) \cup M \cup V$ is a $\tau$-clopen decreasing subset of $X$ containing $v$ but not $u$. This shows that $\Phi(W)$ is a Priestley space.

To prove that $\Phi(W)$ is the dual of a $p$-algebra from ${\underset{\sim}{3}}_{3}$ we need to show that $[V)$ is $\tau$-clopen for every $\tau$-clopen decreasing $V \subseteq X$. If $V \cap C=\emptyset$ then also $V \cap(Y \cup C)=\emptyset$; hence $V$, and thus also $(V]$, is a finite subset of the discrete space $X \backslash(Y \cup C)$. If $I=\left\{i \in 3: c_{i} \in V\right\}$ is nonvoid then $[V]$ consists of $Y$ together with all but finitely many elements of $u\left(A_{i}: i \in I\right) \cup T_{0} \cup T_{1}$ and only finitely many members of each $A_{j}$ with $j \in 3 \backslash I$. Hence $[V)$ is clopen as was to be shown.

For any morphism $f$ of $\underset{\sim}{T} 2$ define $\Phi(f)$ as the extension of $f$ by the identity mapping of $X \backslash Y=M U(T \backslash\{C\})$. It is clear that $\Phi$ is a well-defined one-to-one functor of $\underset{\sim}{T_{2}}$ into the category of $p$-spaces dual to algebras in $\underset{\sim}{B}$, and that $\Phi(f)$ is nonconstant for any morphism $f$ of ${\underset{\sim}{\sim}}_{2}$. Since $M$ is the countably infinite set of minimal elements of each $\Phi(W)$, there are only countably many constant homomorphisms between algebras represented by these spaces. The proof of Theorem 1.1 will be completed once it is shown that every nonconstant continuous order preserving $p$-map $g: \Phi(W)+\Phi\left(W^{P}\right)$ has the form $g=\Phi(f)$ for some morphism $f: W \rightarrow W^{P}$ in $\underset{\sim}{T}$.

The nonconstant $p$-map $g$ maps the minimal set $M$ of $\Phi(W)$ into the minimal set $M$ of $\Phi\left(W^{9}\right)$. Since $\{u, v, w\} \subseteq M$ is the set $\operatorname{Min}(x)$ for some $x \in X$ just when $\{u, v, w\} \in T$, and because the restriction of $g$ to $M$ is a $t$-map, from Proposition 2.5 and (*) it immediately follows that $g$ is the identity mapping on the set $X \backslash(Y \cup C)$. By continuity, $g$ is also the identity mapping on $c$ and $g\left(y_{j}\right)=y_{j}^{3}$ for 
$j \in 2$. Since $Y$ is the set of all elements $x \in X$ for which. $\operatorname{Min}(x)=C$, the restriction $f=g \& Y$ maps $Y$ into $Y^{\prime}$. Recall that the ordered subspace $Y$ of $\Phi(W)$ is homeomorphic and order isomorphic to the ordered space $W \in \underset{\sim}{T}{ }_{2}$. Because it maps the distinguished elements $y_{0}, y_{1}$ of $W$ to the corresponding $y_{0}^{9}, y_{1}^{9} \in W^{\prime}$, the restriction $f=g \vdash W$ is, indeed, a morphism from $W$ into $W^{9}$ in the category $\underset{\sim}{\stackrel{T}{\sim}} ;$ since $g$ is also the identity on $\Phi(W) \backslash Y$, we see that $g=\Phi(f)$ as required. The proof of Theorem 1.1 is now complete.

\section{References}

[1] M. E. Adams, V. Koubek and J. Sichler, "Homomorphisms and endomorphisms in varieties of pseudocomplemented distributive lattices (with applications to Heyting algebras)", Trons. Amer. Math. Soc. 285 (1984), 57-79.

[2] M. E. Adams, V. Koubek and J. Sichler, "Pseudocomplemented distributive lattices with small endomorphism monoids", BuZZ. Austral. Math. Soc. 28 (1983), 305-318.

[3] R. Balbes and Ph. Dwinger, Distributive Lattices, (University of Missouri Press, Columbia, Missouri, 1974).

[4] B. A. Davey and D. Duffus, "Exponentiation and duality", in Ordered Sets, (Reidel, Dordrecht, 1982), 43-95.

[5] V. Koubek, "Infinite image homomorphisms of distributive bounded lattices", in Universal Algebra, (Colloq. Math. Soc. János Bolyai 43, North-Holland, Amsterdam, 1985).

[6] K. B. Lee "Equational classes of distributive pseudocomplemented lattices", Canad. J. Math. 22 (1970), 881-891.

[7] D. Pigozzi and J. Sichler, "Homomorphisms of partial and of complete steiner triple systems and quasigroups", in Universal Algebra and Lattice Theory, (Lecture Notes in Mathematics 1149 , Springer-Verlag, Berlin, 1985, 224-237).

[8] H. A. Priestley, "Representation of distributive lattices by means of ordered Stone spaces", Bull. London Math. Soc. 2 (1970), 186-190.

[9] H. A. Priestley, "The construction of spaces dual to pseudocomplemented distributive lattices", Quart. J. Math. Oxford ser. (2) 26 (1975), 215-228. 
[10] H. A. Priestley, "Ordered sets and duality for distributive lattices", Ann. Discrete Math. 23 (1984), 39-60.

[11] A. Pultr and V. Trnková, Combinatorial, algebraic and topological representations of groups, semigroups and categories, (NorthHolland, Amsterdam, 1980).

[12] P. Ribenboim, "Characterization of the sup-complement in a distributive lattice with last element", Stoma Brasil Math. 2 (1949), 43-49.

Department of Mathematics

State University of New York

New Paltz, NY 12561

U.S.A.

Department of Mathematics

University of Manitoba

Winnipeg, Manitoba

Canada R3T 2N2
MFF KU

Malostranské nám. 25

11800 Praha 1

Czechoslovakia 